\title{
Gray matter networks associated with attention and working memory deficit in ADHD across adolescence and adulthood
}

\author{
Kuaikuai Duan ${ }^{1,2}$, Wenhao Jiang ${ }^{3}$, Kelly Rootes-Murdy ${ }^{3}$, Gido H. Schoenmacker ${ }^{4}$, Alejandro Arias-Vasquez ${ }^{4,5}$, \\ Jan K. Buitelaar (1) ${ }^{4,5}$, Martine Hoogman ${ }^{5}$, Jaap Oosterlaan ${ }^{6,7}$, Pieter J. Hoekstra ${ }^{8}$, Dirk J. Heslenfeld ${ }^{9}$, \\ Catharina A. Hartman ${ }^{8}$, Vince D. Calhoun ${ }^{1,2,3,10}$, Jessica A. Turner $\mathbb{B}^{1,3}$ and Jingyu Liu $\mathbb{D}^{1,10}$
}

\begin{abstract}
Attention-deficit/hyperactivity disorder (ADHD) is a childhood-onset neuropsychiatric disorder and may persist into adulthood. Working memory and attention deficits have been reported to persist from childhood to adulthood. How neuronal underpinnings of deficits differ across adolescence and adulthood is not clear. In this study, we investigated gray matter of two cohorts, 486 adults and 508 adolescents, each including participants from ADHD and healthy controls families. Two cohorts both presented significant attention and working memory deficits in individuals with ADHD. Independent component analysis was applied to the gray matter of each cohort, separately, to extract cohortinherent networks. Then, we identified gray matter networks associated with inattention or working memory in each cohort, and projected them onto the other cohort for comparison. Two components in the inferior, middle/superior frontal regions identified in adults and one component in the insula and inferior frontal region identified in adolescents were significantly associated with working memory in both cohorts. One component in bilateral cerebellar tonsil and culmen identified in adults and one component in left cerebellar region identified in adolescents were significantly associated with inattention in both cohorts. All these components presented a significant or nominal level of gray matter reduction for ADHD participants in adolescents, but only one showed nominal reduction in adults. Our findings suggest although the gray matter reduction of these regions may not be indicative of persistency of $A D H D$, their persistent associations with inattention or working memory indicate an important role of these regions in the mechanism of persistence or remission of the disorder.
\end{abstract}

\section{Introduction}

Attention-deficit/hyperactivity disorder (ADHD) is a childhood-onset neuropsychiatric disorder characterized by inattention, and/or hyperactivity and impulsivity. Recently ADHD persistence into adulthood has been observed in $15-50 \%$ of cases $^{1,2}$, depending on whether or not counting partial remissions $\mathrm{s}^{3,4}$. It is a highly

\footnotetext{
Correspondence: Jingyu Liu (jliu75@gsu.edu)

${ }^{1}$ Tri-institutional Center for Translational Research in Neuroimaging and Data Science (TReNDS), Georgia State University Georgia Institute of Technology and Emory University, Atlanta, GA, USA

${ }^{2}$ School of Electrical and Computer Engineering, Georgia Institute of Technology, Atlanta, GA, USA
}

Full list of author information is available at the end of the article heterogeneous disorder with three clinical presentations recognized in DSM-5 edition ${ }^{5}$, and diverse neurocognitive impairments ${ }^{6,7}$, comorbid disorders, etc ${ }^{6-9}$. Predominant features of adults with ADHD also differ from that of children with ADHD. For instance, adults with ADHD were more affected by inattention than hyperactivity ${ }^{7}$, while children presented symptoms in both inattention and hyperactivity domains. Adult ADHD had a higher rate of comorbidity than children $\mathrm{ADHD}^{10}$, complicating diagnosis ${ }^{11}$. Children with ADHD were affected in more diverse cognitive domains ${ }^{12-19}$ than adults, whose working memory impairments were most frequently documented $^{16,20}$. Working memory deficits in ADHD have 
been consistently reported for both adolescents and adults ${ }^{14,20-22}$. Yet, the underlying neural mechanisms for the different presentations of children and adults with ADHD, as well as consistent presentations, remain elusive. The focus of this study is to investigate gray matter (GM) variation in relation to attention and working memory deficits in both adolescents and adults with $\mathrm{ADHD}^{23,24}$.

Studies of children and/or adolescents have reported GM reduction in widespread brain regions, while the most common effects are in the subcortical regions ${ }^{25-27}$ and cerebellum ${ }^{28}$, followed by regions in the frontal, parietal, temporal cortex ${ }^{29-31}$. Studies of adult patients with ADHD, relatively sparse compared to children, have shown GM alterations in less but more specific brain regions, with the cerebellum and frontal $\operatorname{cortex}^{30,32,33}$ being reported more consistently than subcortical regions ${ }^{25,34,35}$. How these regions GM reduction links to inattention symptom or cognitive deficits is not entirely clear. Castellanos et al. showed that in children and adolescent patients with ADHD, GM volumes of frontal and temporal lobes, caudate, and cerebellum were negatively correlated with the overall score of illness severity, and particularly, attention problems ${ }^{31}$. However, Jacobson et al. showed that GM reduction in the frontal, temporal and parietal lobes were more associated with hyperactive/ impulsive symptoms than inattention ${ }^{36}$. Assisted by functional MRI studies using attentional, working memory, reward and inhibition tasks, these regions have been separated into different networks, fronto-striatal, frontoparietal and fronto-cerebellar networks ${ }^{8}$, interactively subserving functions of reward processing, working memory, orientation of attention, executive control ${ }^{15,37}$, lending support to the brain networks based analyses. Furthermore, the structure and function of the networks change with age $\mathrm{e}^{25,38}$, which promotes direct comparison of the brain networks between children/adolescents and adults with ADHD. Any knowledge gained on the network characteristics and relationship of adolescent and adult ADHD might shed light on the mechanism of persistence or remission of the disorder ${ }^{8,39}$.

As reviewed by Sudre et al., the remission (otherwise persistence) of ADHD can be modeled as three processes, including 'neural normalization', 'neural reorganization', and 'fixed anomaly', which are not exclusive and might occur in different brain networks ${ }^{39}$. In particular, brain networks serving 'top-down' cognition as working memory or attention largely experience neural normalization; i.e., early anomalies in brain structure and function disappear in remitted brains, whereas persistence is linked with persisting neural anomalies ${ }^{39}$. When comparing brain GM networks (i.e., brain regions with similar or related GM variations across participants as explained later) associated with attention or working memory between adolescents and adults with ADHD, we hypothesize persistent brain network associations will present at large. As the brain changes much more dramatically in adolescents than adults, we also hypothesize that GM networks of adolescents and adults will comprise different but overlapping brain regions.

To test our hypotheses, we applied independent component analysis (ICA) to GM of two age groups, adults and adolescents. ICA has been used for various types of data, including both structural ${ }^{40-42}$ and functional MRI data $^{43-45}$ to extract brain networks. When applied to gray matter density maps as we did in this study, it surveys the whole brain without preselection of regions of interest, and utilizes voxels GM variation patterns across participants to group voxels with similar or related patterns into components, forming coherent GM networks. Thus, ICA is a data-driven network-based analysis. This work utilized ICA to extract GM networks of two age groups separately, reflecting inherent GM network configuration of their own. Then we tested GM networks' associations with inattention and working memory in their own group, and cross evaluated for the other group. Our previous study has analyzed the adult group and reported five GM networks of interest ${ }^{24}$. Here we extended into the adolescent group and focused on the comparison and relationship between the two age groups. The findings of this study will answer whether persistent brain networks underlie persistent attention and working memory deficits in ADHD.

\section{Participants and methods \\ Participants}

This study analyzed data from two ADHD projects: the Dutch chapter of the International Multicentre persistent ADHD genetics CollaboraTion (IMpACT) consortium $^{13,46}$, and the NeuroIMAGE project ${ }^{47}$. The IMpACT project recruited adult participants with ADHD and healthy controls, while the NeuroIMAGE project recruited participants from ADHD families (including probands and siblings) and healthy controls in childhood and then followed them up. Data used here were from the 1st MRI scan with some participants in adolescence and some in adulthood. The inclusion and exclusion criteria were described in the original papers ${ }^{46,47}$. In brief, the IMpACT ADHD participants met DSM-IV-TR criteria for ADHD in adulthood as well as childhood retrospectively. The NeuroIMAGE participants met DSM-IVTR criteria for children or adult ADHD, and adult participants also had a formal and research diagnosis in childhood. All participants had IQ $\geq 70$, no diagnosis of autism, epilepsy, general learning difficulties, brain disorders, and genetic disorders (such as Fragile X syndrome or Down syndrome). The Dutch chapter of IMpACT study was approved by the regional ethics committee 
(Centrale Commissie Mensgebonden Onderzoek: CMO Regio Arnhem-Nijmegen; Protocol number III.04.0403). Written informed consent was obtained from all participants. The NeuroIMAGE study was approved by the regional ethics committee (Centrale Commissie Mensgebonden Onderzoek: CMO Regio Arnhem-Nijmegen; 2008/163; ABR: NL23894.091.08) and the medical ethical committee of the VU University Medical Center. Written informed consent for every participant was obtained. For children 12-18 years old, both parents and children gave consent. For children younger than 12, parents gave consent for their children.

In order to compare adults with adolescents, the participants of IMpACT and NeuroIMAGE projects were regrouped into adult samples $(N=486$, age $\geq 18$, including participants of IMpACT and NeuroIMAGE) and adolescent samples $(N=508,7<$ age $<18$, part of NeuroIMAGE; we named this group as adolescents since 436 participants were older than 12). A summary of participant demographics is shown in Table 1. Concerning the large age range in each group, we also tested subsets of participants, which included 427 adults $(18 \leq$ age $<40$, control $/$ case $/$ sibling $=139 / 192 / 96, \quad$ female $/ \mathrm{male}=212 /$ $215)$ and 436 adolescents $(12<$ age $<18$, female/male $=$ $177 / 259$, controls/cases/siblings $=137 / 174 / 125)$.

\section{ADHD symptoms and working memory scores}

Two ADHD domains, inattention and hyperactivity/ impulsivity, were assessed for all participants. NeuroIMAGE used the Schedule for Affective Disorders and Schizophrenia - present and lifetime version, and Conners Teacher Rating Scale-1997 Revised Version: Long Form. IMpACT used the Diagnostic Interview for Adult ADHD. The symptom scores for both domains were counted following the 18 DSM-IV questions, with the score in each domain ranging from 0 to 9 . Healthy controls had scores less than 2 in either domain. Unaffected siblings from patient families had scores fewer than 5 or 6 in either domain for adult or adolescent participants, respectively. Both IMpACT and NeuroIMAGE projects conducted WAIS Digit Span test ${ }^{48}$. We utilized maximum forward and maximum backward scores to gauge working memory capacity (other measures of working memory, such as visual-spatial working memory test for functional MRI, were only available for part of participants. Thus, we only focused on digit span test). In the adult group, participants with ADHD had significantly lower scores in both forward and backward tests than controls (forward: $t$-test $p=4.36 \times 10^{-4}$, Cohen's $\mathrm{d}=0.47$; backward: $t$-test $p=3.76 \times 10^{-5}$, Cohen's $\left.\mathrm{d}=0.55\right)$. Similarly, adolescent participants with ADHD also presented significantly lower forward and backward scores (forward: Cohen's $\mathrm{d}=0.37, t$-test $p=1.57 \times 10^{-4}$; backward: Cohen's $\mathrm{d}=$ $0.44, t$-test $p=5.58 \times 10^{-6}$ ).

\section{Imaging data acquisition and processing}

T1-weighted MRI images were acquired with three 1.5T scanners with closely matched settings. Thorough quality control was applied to the imaging data as previously described, including selecting the better one from the two sessions. MRI quality indexes (coefficient of joint variation, contrast-to-noise ratio, entropy focus criterion ${ }^{49}$ ) were generated for further quality check. All good quality images were segmented into six types of tissues using Statistical Parametric Mapping 12 (SPM12, http://www.fil. ion.ucl.ac.uk/spm/software/spm12/), followed by normalization to the Montreal Neurological Institute space, modulated, and smoothed with a $6 \times 6 \times 6 \mathrm{~mm}^{3}$ Gaussian kernel. The only difference during the preprocessing of adult and adolescent data was the tissue probability map templates used for segmentation, where SPM12 templates were used for adults and age-specific tissue map templates generated by TOM tool ${ }^{50}$ were for adolescents. Further analyses on segmented GM images were done separately

Table 1 Demographic and assessment information of participants.

\begin{tabular}{|c|c|c|c|c|c|c|}
\hline & \multicolumn{3}{|c|}{ Adults (age 18-63) } & \multicolumn{3}{|c|}{ Adolescents (age 7-17) } \\
\hline & ADHD & Siblings & Controls & ADHD & Siblings & Controls \\
\hline $\mathrm{N} /$ male/medicated & 214/123/105 & $96 / 49 / 0$ & $176 / 51 / 0$ & 210/129/121 & $140 / 59 / 12$ & 158/93/2 \\
\hline Age & $25.35 \pm 8.56$ & $21.41 \pm 2.34$ & $28.93 \pm 11.79$ & $14.61 \pm 2.41$ & $14.80 \pm 2.07$ & $14.56 \pm 2.17$ \\
\hline $\mathrm{IA}$ & $7.23 \pm 1.66$ & $1.60 \pm 1.97$ & $0.56 \pm 1.24$ & $7.33 \pm 1.71$ & $1.34 \pm 1.96$ & $0.77 \pm 1.67$ \\
\hline $\mathrm{HI}$ & $5.79 \pm 2.38$ & $1.48 \pm 1.65$ & $0.70 \pm 1.12$ & $5.99 \pm 2.39$ & $0.99 \pm 1.57$ & $0.36 \pm 1.10$ \\
\hline Digit span forward & $7.94 \pm 1.79$ & $8.58 \pm 1.67$ & $8.76 \pm 1.73$ & $8.86 \pm 1.96$ & $9.39 \pm 1.88$ & $9.58 \pm 1.94$ \\
\hline Digit span backward & $5.09 \pm 1.74$ & $5.99 \pm 1.69$ & $6.12 \pm 2.02$ & $6.45 \pm 2.25$ & $6.63 \pm 2.36$ & $7.39 \pm 2.05$ \\
\hline Scan* & $58 / 68 / 88$ & $40 / 56 / 0$ & $47 / 32 / 97$ & $95 / 115 / 0$ & $76 / 64 / 0$ & $95 / 115 / 0$ \\
\hline
\end{tabular}

IA inattention, $H$ I hyperactivity/impulsivity, Scan* NeurolMAGE 1/ NeurolMAGE 2/ IMpACT (Dutch). 
for adults and adolescents, including selecting individual maps that had correlations with the group mean GM hinger than 0.8 , generating a GM mask for each group to include voxels with group mean GM volume larger than 0.2 , and regressing out effects of age, sex, and site using a linear regression model for each included voxel. The GM mask of adults was slightly different from adolescents, and comparison analyses were applied only to the common voxels (441,258 voxels).

\section{GM components in adults and adolescents}

In the ICA model, $\mathbf{X}=\mathbf{A S}, \mathbf{X}$ is the GM data matrix, $\mathbf{S}$ is the component matrix, and $\mathbf{A}$ is the loading matrix. Each row of S shows weights of individual voxels in one component. Each column of $\mathbf{A}$ shows expression or loadings of individual subjects for one component. A component reflects coherent GM variations within a brain network with the flexibility to go beyond the known boundaries of brain anatomy ${ }^{42}$. This approach not only reduces multiple comparisons required in whole-brain analyses, but also dissects the brain into structurally independent networks. For example, large areas of the frontal lobe may all relate to working memory, but different subregions may relate to working memory in different ways. Whole-brain voxelwise analysis based on association significance would not be able to separate them, while ICA separates the brain into independent networks first and then tests each network's properties. ICA toolbox is available in https:// trendscenter.org/software/gift.

As reported in our previous study when ICA was applied on adult GM data ${ }^{24}$, five components out of 22 were significantly related to inattention, working memory deficit, or ADHD diagnosis in adult participants (supplementary text). To test how these structural alterations present in adolescents, we projected these GM components onto the adolescent data. Specifically, using the components derived from adults $\mathrm{S}$, we applied $\mathbf{A}_{\mathbf{c}}=$ $\mathbf{X}_{\mathbf{c}} \mathbf{S}^{-1}$, where $\mathbf{X}_{\mathbf{c}}$ was the adolescent GM data, yielding the projected loading matrix $\mathbf{A}_{\mathbf{c}} . \mathbf{A}_{\mathbf{c}}$ showed how the adult brain components were expressed in adolescents, on which we tested the associations with diagnosis, symptoms, and working memory in adolescents. Vice versa, we applied ICA to the adolescent GM data in the same manner. Twenty components were extracted and tested for associations with diagnosis, symptoms, and working memory. For the components with significant associations, we projected them onto the adult data, followed by association tests with diagnosis, symptom and working memory in adults.

\section{Statistical analyses}

A linear mixed model with family structure as a random effect and diagnosis as a fixed effect was applied onto GM loadings of cases and controls to test case vs. control differences (age, sex, and site had been regressed out voxel-wise in the preprocess). Similarly, linear mixed models with symptom score or working memory score as a dependent variable, GM loading, age, and sex as fixed effects and family as a random effect were applied to test GM association with symptoms or working memory using all participants (cases, control, and siblings) in the group. Given that medication can affect both brain and behavior, we first compared GM loadings between medicated cases and unmedicated cases. If there was a significant difference $(p<0.05)$, we added medication status (used vs. not used) as a fixed effect in the linear models. Comorbidity with major depression and anxiety were also tested by adding them separately as a covariate into the linear models. We did not control for IQ, because it can remove ADHD related variance ${ }^{51}$. Note that for both directions of cross-evaluation (components discovered in adult$\mathrm{s} \rightarrow$ projection to adolescents for evaluation, and components discovered in adolescents $\rightarrow$ projection to adults for evaluation), we applied false discovery rate (FDR) $p<0.05$ to control for multiple comparisons (22 components for adults and 20 components for adolescents, respectively) on the discovery results, and uncorrected $p<0.05$ for evaluation of the projected results.

Given the large age range of participants, particularly in adults, we performed additional analyses to enhance the stability of test results. First, we selected participants with age between 18 and 40 years old, performed a separate ICA and compared the resultant components with those derived from full adult samples reported in ref. ${ }^{24}$. Second, we replicated association tests with working memory and inattention for the identified components using homogenous subset of adults (18-40 years old) and adolescents (12-17 years old). Giedd et al. have reported GM in the frontal lobe increased during preadolescence with a maximum size occurring at the age of 12.1 years for males and 11.0 years for females, followed by a decline during postadolescence $^{52}$. So, we chose 12-17 years old as a homogenous adolescent group.

\section{Results}

In the previous study we have reported five GM components in adults significantly associated with working memory, inattention, or diagnosis. The analyses and results of our previous study are summarized in the supplementary text. The five components are plotted in Fig. 1 (Independent Component (IC) 1-5). Using the homogenous subgroup 427 participants' GM data, a separate ICA extracted highly similar components. The correlations between subgroup components and fullsample components were $0.97,0.85,0.95,0.96$, and 0.97 for the five components, respectively. The correlations between loading coefficients of the components were 0.98 , $0.96,0.99,0.98$, and 0.98 . The validity of the five 


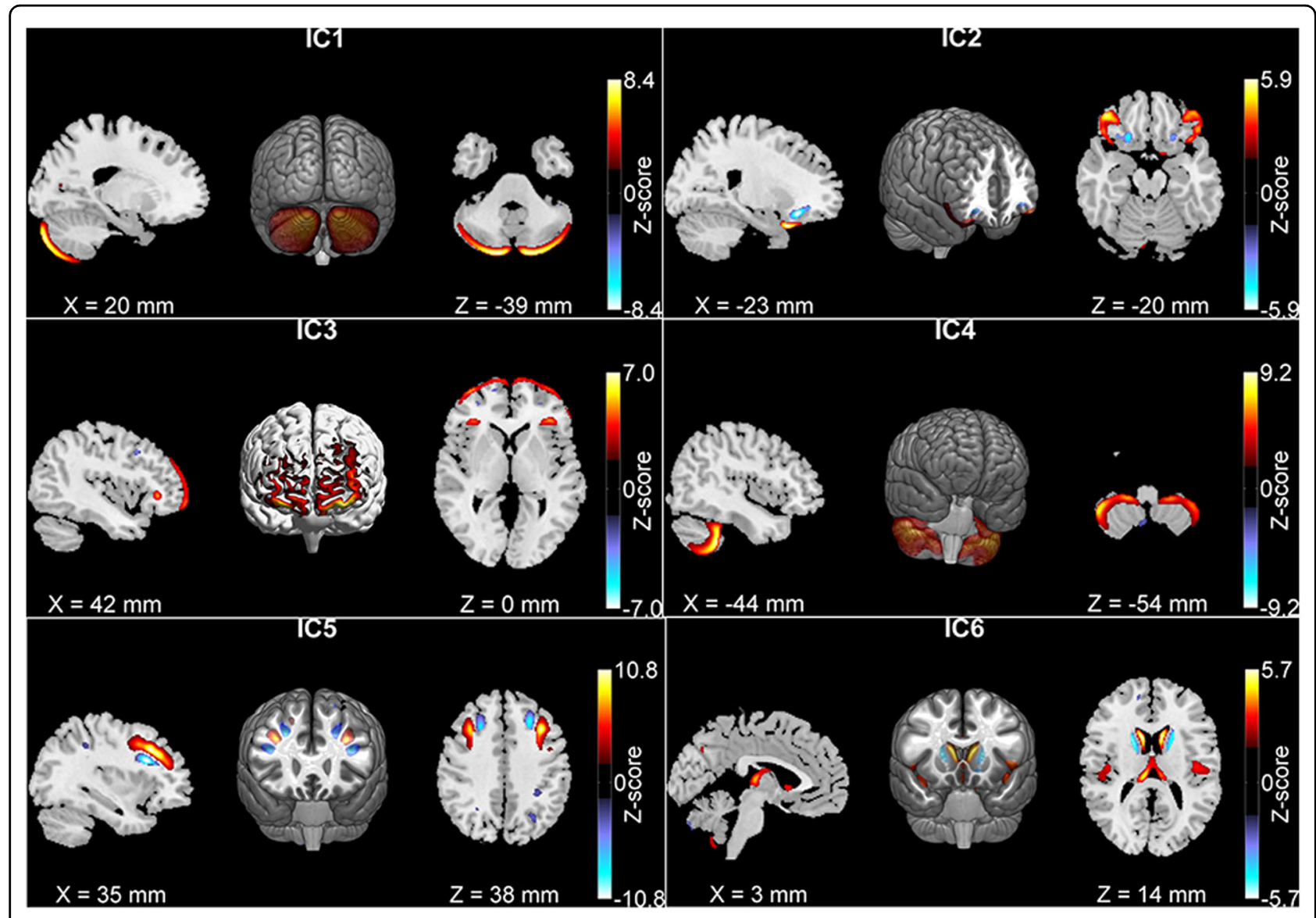

Fig. 1 The six gray matter components identified in adults. In adults, ICs 1-3 were associated with working memory, IC 4 was associated with inattention, IC 5 showed gray matter reduction in patients, and IC 6 included the caudate nucleus.

components has been provided before ${ }^{24}$. Additionally, we tested possible confounding effects from three MRI quality metrics (coefficient of joint variation, contrast-tonoise ratio, entropy focus criterion), using two regression models: (1) working memory or symptom $=\mathrm{GM}$ loading + age + gender + family (random effect) + three MRI quality metrics, and (2) GM loading = case/control + family + three MRI quality metrics. The new test results agreed with previously reported association results (components were significantly associated with working memory, inhibition or case-control status with $p<0.003$ ). Given the high consistency, we continued our analyses with the full-sample derived components previously reported.

After we projected the five components into adolescents, their associations with working memory and inattention in both adults and adolescents were listed in Table 2A. In adults, Components 1-4 showed no GM differences between medicated and unmedicated cases $(p>0.05)$, while Component 5 showed a significant medication effect. Thus, the association results of Component 5 were computed with a linear model controlling for medication. In adolescents, Components 1-4 showed significant GM increases in medicated cases compared to unmedicated cases $(p<0.05)$. Thus, their association results were from linear models controlling for medication. Component 5 showed no medication effect and no case vs. control difference. No significant comorbidity effect was observed for these components in both adults and adolescents.

Across adults and adolescents, three GM components (ICs 2-4 in Table 2A) showed consistent associations with either working memory or inattention. Component 2 , the inferior frontal gyrus, and Component 3 , the superior and middle frontal gyri, were positively associated with working memory in both adults and adolescents. More GM volume was associated with higher (better) working memory scores in all participants. Component 4, the cerebellar tonsil and culmen, was negatively associated with inattentive symptom in both adults and adolescents, where lower GM volume was associated with higher (worse) inattentive score. The effect size was computed as the percentage of variance explained, R-square, listed in Table 2. In general, the 
Table 2 Association results of gray matter components identified in adults and adolescents.

\begin{tabular}{|c|c|c|c|}
\hline & & Association in adults & Association in adolescents \\
\hline \multirow[t]{2}{*}{ IC 1} & Case vs. control & n.s. & $p=2.63 \times 10^{-2} ;$ reduction, $\mathrm{d}=-0.44$ \\
\hline & association & $\begin{array}{l}\text { With working memory (forward) } p=1.4 \times 10^{-4} \\
\text { positive, } \mathrm{R}^{2}=3.08 \%\end{array}$ & With working memory (forward) n.s. \\
\hline \multirow[t]{2}{*}{ IC 2} & Case vs. control & n.s. & $p=1.56 \times 10^{-4} ;$ reduction, $d=-0.77$ \\
\hline & association $^{\text {a }}$ & $\begin{array}{l}\text { With working memory (forward) } p=2.68 \times 10^{-3} \text {; } \\
\text { positive, } R^{2}=1.90 \%\end{array}$ & $\begin{array}{l}\text { With working memory (forward) } p=2.72 \times 10^{-2} \\
\text { positive, } \mathrm{R}^{2}=0.88 \%\end{array}$ \\
\hline \multirow[t]{2}{*}{$1 \mathrm{C} 3$} & Case vs. control & n.s. & $p=4.51 \times 10^{-3} ;$ reduction, $\mathrm{d}=-0.72$ \\
\hline & association $^{\text {a }}$ & $\begin{array}{l}\text { With working memory (backward) } p=1.66 \times 10^{-3} \text {; } \\
\text { positive, } \mathrm{R}^{2}=2.05 \%\end{array}$ & $\begin{array}{l}\text { With working memory (forward) } p=1.33 \times 10^{-2} \\
\text { positive, } \mathrm{R}^{2}=1.13 \%\end{array}$ \\
\hline \multirow[t]{2}{*}{ IC 4} & Case vs. control & $p=1.04 \times 10^{-2} ;$ reduction, $d=-0.59$ & $p=1.35 \times 10^{-2}$, reduction, $d=-0.47$ \\
\hline & association $^{a}$ & $\begin{array}{l}\text { With inattentive symptom } p=2.26 \times 10^{-3} \text {, negative, } \\
R^{2}=1.88 \%\end{array}$ & $\begin{array}{l}\text { with inattentive symptom } p=3.04 \times 10^{-2} \text {, negative, } \\
R^{2}=0.61 \%\end{array}$ \\
\hline \multirow[t]{2}{*}{ IC 5} & Case vs. control & $p=5.56 \times 10^{-6} ;$ reduction, $d=-0.74$ & n.s. \\
\hline & association & n.s. & n.s. \\
\hline \multirow[t]{2}{*}{ IC 6} & Case vs. control & n.s. & $p=3.34 \times 10^{-2}$, reduction, $d=-0.35$ \\
\hline & association & n.s. & $\begin{array}{l}\text { With working memory (forward), } p=7.86 \times 10^{-3} \text {, positive, } \\
\mathrm{R}^{2}=1.34 \%\end{array}$ \\
\hline
\end{tabular}

(B) GM components identified in the adolescent group

\begin{tabular}{llll}
\hline & & Association in adults & Association in adolescents \\
\hline IC- & Case vs. control & n.s. & $p=5.37 \times 10^{-4}$; reduction, $\mathrm{d}=-0.73$ \\
A & association $^{a}$ & With inattentive symptom $p=2.15 \times 10^{-2} ;$ & With inattentive symptom $p=2.01 \times 10^{-3}$, negative, \\
& & negative, $\mathrm{R}^{2}=1.09 \%$, & $\mathrm{R}^{2}=1.19 \%$ \\
IC- & Case vs. control & n.s. & $p=1.78 \times 10^{-3} ;$ reduction, $\mathrm{d}=-0.58$ \\
B & association $^{\text {a }}$ & With working memory (forward) $p=4.58 \times 10^{-3} ;$ & With working memory (forward) $p=4.02 \times 10^{-3} ;$ \\
& & positive, $\mathrm{R}^{2}=1.71 \%$ & positive, $\mathrm{R}^{2}=1.50 \%$
\end{tabular}

$\mathrm{d}$ denotes the Cohen's $\mathrm{d}$ value. Results in bold font are significant, where the significance in discovery data were set to pass FDR $p<0.05$, and the significance in verification data after projection was set to pass uncorrected $p<0.05$. $p$-values shown in the table are uncorrected. $\mathrm{R}^{2}$ indicates variance of working memory or inattention score explained by the IC.

n.s. not significant.

${ }^{\mathrm{a}}$ Indicates consistent associations across adolescents and adults.

variance explained by each GM component is small (around 2\%), but significant. In addition to these significant associations, it is noteworthy that adolescent patients showed nominal GM reduction in four of the five components $(p<0.05)$, while adult patients showed GM reduction in two components (one passed FDR and one had $p<0.05)$.

Twenty components were extracted from the adolescent data of which twelve were highly similar to those in adults $(\mathrm{r} \geq 0.5$, see Supplementary Table 1). Two components (Fig. 2) showed significant GM reduction in patients and were also significantly associated with inattention or working memory in adolescents. For these two components, medicated cases had increased GM volumes compared to unmedicated cases $\left(p=4.64 \times 10^{-3}\right.$ and $p=$ $3.95 \times 10^{-2}$, respectively). After projection to adults, no medication effects were observed. Thus, medication effect was controlled only for adolescents. As shown in Fig. 2, Component A comprised left hemisphere, cerebellar tonsil and culmen, lingual gyrus, and cuneus. Component B comprised bilateral insula, inferior frontal, superior temporal gyri, and caudate nucleus. Table 2B lists out associations of the two components with working memory, inattention, and diagnosis in both age groups. GM 


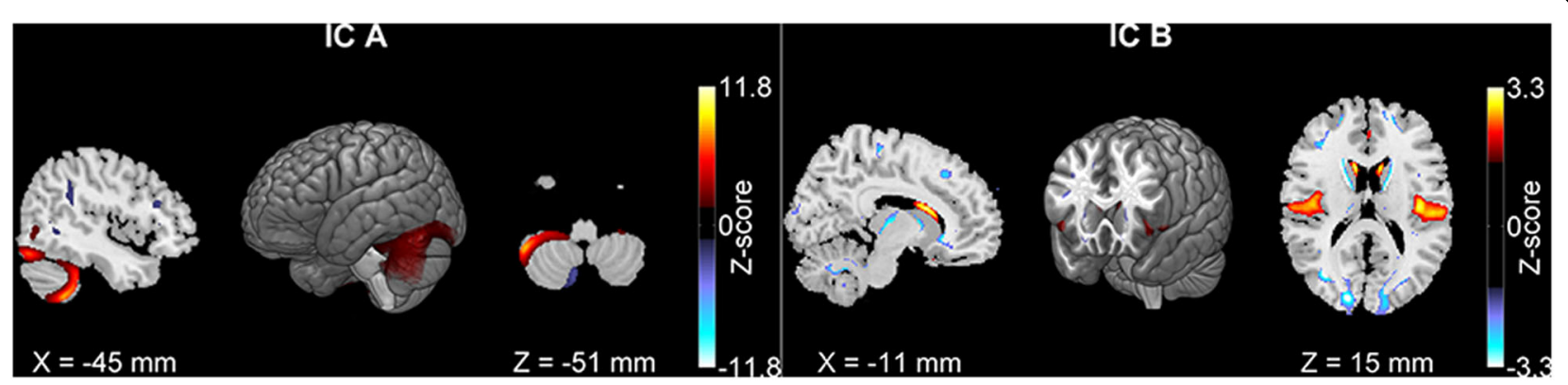

Fig. 2 The two gray matter components identified in adolescents. IC-A was associated with inattention and IC-B was associated with working memory. Both components had gray matter reduction in ADHD patients.

volume of Component A was negatively associated with inattentive symptom in adolescents and adults and showed a significant reduction in adolescents with ADHD. GM volume of Component B was positively associated with working memory in both groups and showed a significant reduction in adolescents with ADHD. The effect sizes, R-squares, are listed in Table 2B. No significant comorbidity effect was observed for the components in both groups.

Literatures have shown that subcortical regions like the caudate nucleus and putamen were significantly reduced in ADHD patients and the reduction seemed to diminish over time from childhood to adulthood ${ }^{25,38,53}$. Functionally fronto-striatal network ${ }^{54}$ is a key component supporting executive function including attention and working memory ${ }^{55}$, and its alterations in $\mathrm{ADHD}^{34,56}$ is hypothesized as a core of one ADHD etiology model ${ }^{6}$. Given the relevance, we specifically picked components including the caudate nucleus: one adult component, (Component 6 in Fig. 1, comprising caudate nucleus, superior temporal and insula), and one adolescent component (Component B in Fig. 2). These two components were highly correlated $(\mathrm{r}=0.58, p<1 \mathrm{e}-16)$. But the adolescent component had more areas in insula and inferior frontal gyrus and less in the caudate nucleus relative to the adult one. As listed in Table 2A, Row IC 6 and Table $2 \mathrm{~B}$, Row IC-B, the adult component showed no associations in adults but nominal associations with working memory and diagnosis in adolescents $(p<0.05)$, while the adolescent Component B showed consistent significant associations with working memory in both groups and significant GM reduction in adolescents.

Due to the large age range of each group, we replicated association tests for these eight components using relatively homogenous group settings (adolescents: 12-17 years old with 436 participants; young adults: $18-40$ years old with 427 participants). The results (Supplementary Table s2) were largely consistent with those derived using all participants. All associations reported in Table S2 except two presented consistent significant results. The two in discrepancy were where subsamples could not report associations with $p<0.05$.

We also compared GM volumes of the unaffected siblings with healthy controls and patients with ADHD. Figure 3 plots GM loadings of each diagnostic group, each age group and each component. In adolescents, seven components (except IC 5) had some levels of GM reduction in patients with ADHD (significant or nominal, see Table 2). Siblings had more GM volume than patients but less than controls in five components. However, most of the differences were not statistically meaningful $(p>$ 0.05). In adults, siblings presented no clear patterns. Similar results were observed when we examined only unmedicated patients (see results of unmedicated cases, siblings, and controls in Supplementary Fig. s1).

\section{Discussions and conclusions}

Aiming at the GM alterations of ADHD patients in adolescence and adulthood, we compared GM patterns of the two age groups extracted by data-driven whole-brain ICA approaches. GM networks derived by ICA are sensitive to data characteristics (i.e., voxels grouped together as a component based on gray matter density variation in children may be very different from voxels grouped together as a component in adults). In the 20 components extracted from adolescents, 12 were highly similar to those in adults, supporting continuation of brain structural segmentations. The differences between similar components and the totally different components likely indicate developmental effects on similar regions of brain, as well as disorder progress effect, to form the unique constellation to each group. To ensure fair comparisons between the two age groups, we conducted the network discovery-and-verification two-step approach in both directions: from adults to adolescents and from adolescents to adults. Additionally, adolescent patients with ADHD will inevitably include both those likely to persist and those likely to remit. This mixture will most likely contribute to the different GM patterns between the adolescent group and the adult group that included only 

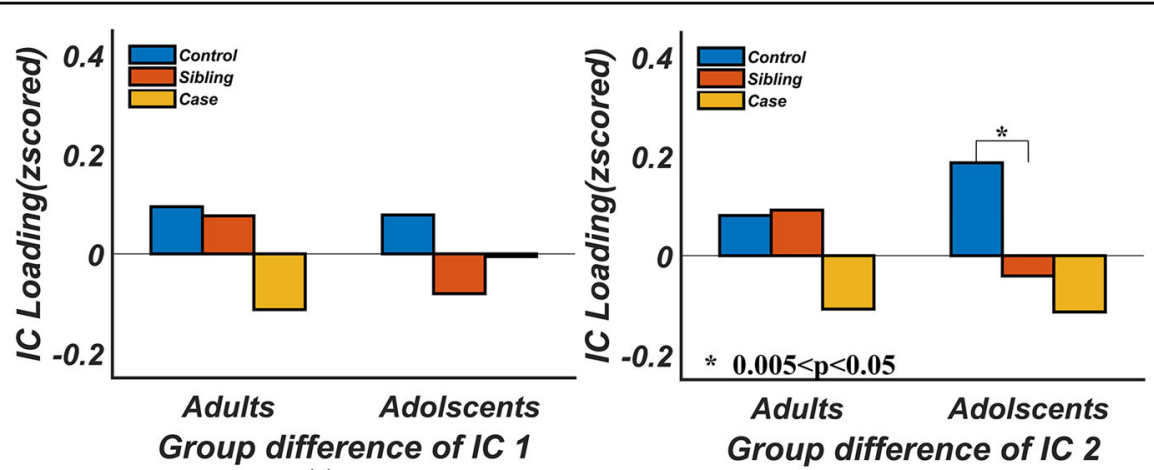

(a)

(b)
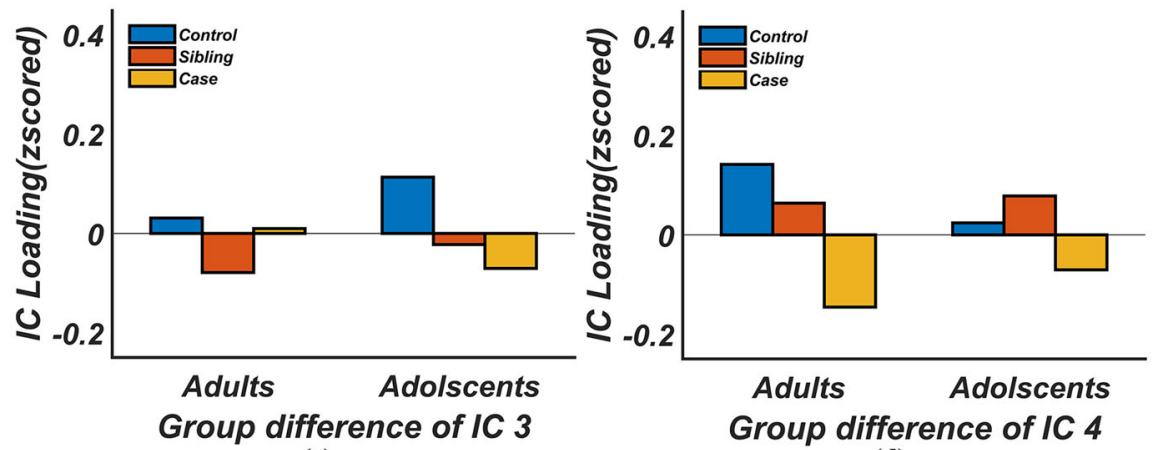

(c)

(d)
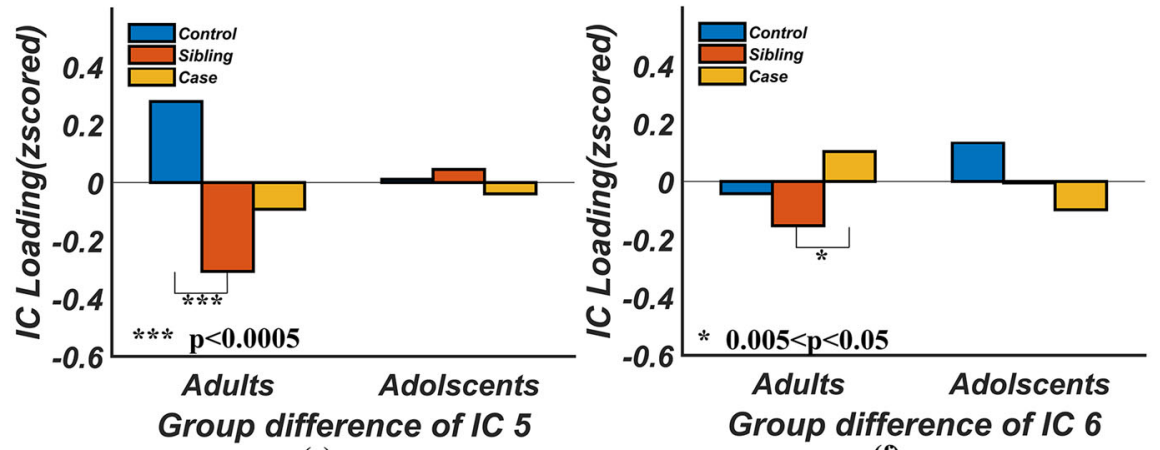

(e)

(f)

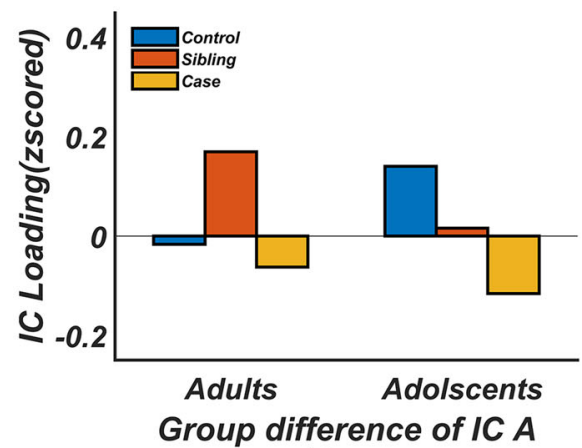

(g)

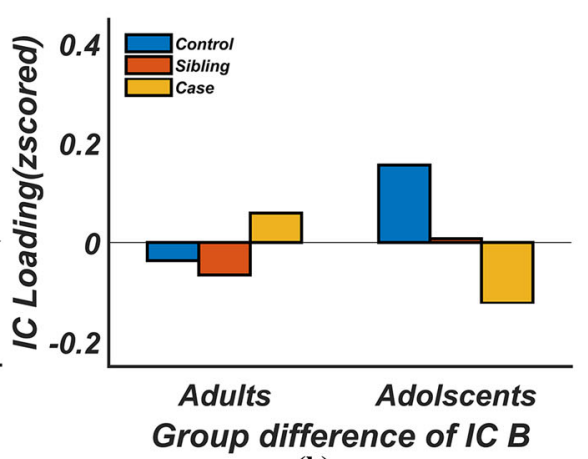

(h)

Fig. 3 Comparison of gray matter volumes in unaffected siblings against those in controls and patients. $P$-values for the comparisons between cases and controls were listed in Table 2, and not plotted here for the clarity of the figure. Only the significant results for comparisons involving siblings $(p<0.05)$ are plotted. 
persistent ADHD patients. Thus, our discussion focuses on consistent findings between the two age groups.

For the four components associated with working memory (three adult components and one adolescent component passing multiple comparison correction), three components showed consistent associations across childhood to adulthood, including the superior, middle, and inferior frontal regions (adult IC 2-3), and the insula, inferior frontal, superior temporal gyri and caudate (adolescent IC-B). The prefrontal regions are well documented for their role in working memory as a central executive point $^{57}$, involving integrating sensory information, allocating neural resources, maintaining and shifting attention, and updating and manipulating information ${ }^{58-60}$. Better working memory performance has been found to correlate with greater GM volume in widespread brain areas, including the superior, middle, and inferior frontal gyri $^{61,62}$. Interestingly, a recent large sample study (1336 young adults) on the relationship between regional GM and cognition reported that the strongest association between GM and working memory was in the insula, which was the only region significantly associated with working memory after controlling for total brain volume ${ }^{62}$. The longitudinal study led by Biederman et al. suggested cognitive impairments in ADHD originating in childhood persisted into adulthood ${ }^{63}$. Our results suggest that not only working memory deficits persisted from childhood and adulthood, but also did the neural correlates of working memory deficits, highlighting the frontal regions and insula.

Two components (adult component 4 and adolescent component A) were significantly associated with inattention across both age groups, with more GM volume related to lower inattentive symptom scores. Component 4 mainly consisted of bilateral cerebellar tonsil and culmen, and Component A consisted of left hemisphere cerebellar tonsil and culmen, lingual gyrus, and cuneus. These two components were spatially similar with a correlation of 0.49 , overlapping mostly in left hemisphere cerebellar regions. The differences likely reflect different developmental trajectories of left and right cerebellar hemispheres in adolescence. Both adult and adolescent components support the involvement of the cerebellum in ADHD, consistent with repeatedly reported cerebellum volume reduction as the most stable brain alterations observed in ADHD patients s, $30,31,64-66^{\text {. }}$

The role of the cerebellum in cognitive function has been recognized ${ }^{67,68}$, supported by cognitive impairment in patients with cerebellum lesions ${ }^{69}$, prevalent activation during cognitive functions ${ }^{70}$, anatomic connections between the cerebellum and cortical regions ${ }^{71,72}$, and functional co-organization with cortical regions ${ }^{73,74}$. In ADHD patients, the functional impact of cerebellum GM alteration is less studied, with the potential to affect neurological soft signs ${ }^{75,76}$, attention process as part of executive function ${ }^{68}$, or both as these two could be linked ${ }^{77,78}$. Our findings add to the literature and emphasize a specific role of the cerebellum in the attention process, and its persistence from childhood to adulthood.

A detailed look at the components including the caudate nucleus appeared to show that some caudate areas were integrated together with the insula, inferior frontal, and superior temporal regions for both adults and adolescents. An additional analysis using GM data without voxel-wise regression of age effect extracted a more complete/dominant caudate component (Supplementary Fig. s2). The spatial difference of the components with and without voxel-wise age regression indicates that age had a significant role and carried a large proportion of variance in the caudate nucleus. In fact, one previous study using NeuroIMAGE data has reported developmentally sensitive caudate alterations in relation to $\mathrm{ADHD}^{38}$ : caudate volume reduction in younger ADHD patients (age 8-15), not in middle age patients (age 15-22), and reversed in older patients (age 22-30). Our age grouping and processing approach resulted in the components that included partial caudate and interrelated insula ${ }^{79}$ and cortical regions ${ }^{80}$, not a typical whole caudate region. Nevertheless, the comparison of the two components that include the caudate nucleus advocates the role of the insula and inferior frontal regions in working memory processes, as more consistent and more significant associations with working memory were observed for the component with more insula and inferior frontal region.

In contrast to the consistent results discussed above, we found that GM reductions observed in adolescent ADHD patients were largely not replicated in the adult group. The two components, IC-A and IC-B showed significant GM reductions in adolescent patients (passing multiple comparison correction), but not in adults. The other five components ICs $1-4$ and 6 showed nominal $(p<0.05)$ GM reductions in adolescent patients, and only one component (IC 4) showed comparable levels of reduction in adults. Moreover, we also observed more prevalent medication effects on GM in adolescents than in adults. Among the seven components with nominal or significant GM reduction in adolescents, six showed that medication mitigated the reduction in patients. Altogether these findings agree with previous studies reporting GM reduction in ADHD patients diminished with age, particularly in subcortical regions ${ }^{25}$. Our results expand the affected regions into the frontal and cerebellum regions. One note is that medication could, at least, partially contribute to the observed diminished GM reduction with age.

Comparison of GM volumes of siblings against cases and controls in Fig. 3 produced mostly no significant differences. We think this might be due to two reasons. 
One is that our sample size is too small to reach a significant conclusion. The other, which is more likely, is the heterogeneity of the sibling group, whose ADHD symptom scores ranged from 0 to $5 / 6$ (adult/children, respectively). Studies have shown participants with symptom scores higher than 2 could be considered as subthreshold $\mathrm{ADHD}^{81,82}$, and they were at greater risk for negative outcomes in cognitive domains ${ }^{83}$. Little is known about subthreshold ADHD for which more investigations are needed.

Overall, through direct comparisons of GM components in both adolescents and adults, our findings suggest that brain regions associated with deficits in working memory and attention in ADHD patients persist from childhood into adulthood. These regions include the inferior/superior/middle frontal gyri and the insula for working memory, and the cerebellar tonsil and culmen for attention. In contrast, due to developmental and disorder progress differences between adolescents and adults, how these regions are grouped into independent networks could be different, as illustrated in GM components. And GM reduction of these regions observed in younger patients (adolescents) largely diminishes in adulthood. This phenomenon, beyond subcortical regions, could be partially contributable to medication and development (including disorder progress). Although the GM reduction of these regions is not indicative of persistence of impairments, their persistent associations with inattention or working memory suggest an important role of these regions in the mechanism of persistence or remission of the disorder. ADHD has been described as a disorder of executive function ${ }^{11}$, in particular working memory alterations have been viewed as a core neurocognitive deficit of ADHD, responsible for recognition of external stimuli, and leading to inattention symptoms. ${ }^{6}$ We speculate that the function and structure of frontalinsula regions associated with working memory and cerebellum with inattention might have different refined network configurations in children and adults, and in individuals with or without ADHD, and the differences of these networks in terms of spatial shape, GM density, functional intensity and connectivity could help to delineate remittance and persistence of ADHD. Future longitudinal investigations of the dynamics of these regions would be warranted.

Findings of this study should be interpreted with consideration of the following limitations. First, the age ranges of both groups are large. To mitigate the heterogeneity, we have tested relatively homogenous age groups (adolescents of 12-17 years old and adults of $18-40$ years old). Similar findings as discussed above were observed. Second, our analytical approaches focus on the comparison of two age groups with age regression specific to each group. This approach leads to common patterns across the age range within each group, suitable for group comparisons. However, the dynamic developmental trajectories, like the one in the caudate nucleus, are missed. Third, ICA segments brain regions with coherent patterns together, including those with opposite patterns as shown in blue in Figs. 1 and 2. The blue regions were smaller and contributed with lower weights than the positive regions in red. They showed opposite effects to the positive regions as discussed above. The interpretation of these regions is limited and further specific studies to verify these regions effect are necessary. Forth, WAIS digit span was used to probe working memory. It has limited the testing range and is not as good as the visual-spatial working memory test. However, our data clearly showed working memory deficits in individuals with ADHD. Future analyses with more sensitive measures could verify and refine our findings. Finally, even though we have tried to control for confounding factors like medication and comorbidity (depression and anxiety) in the analyses, other factors potentially affecting ADHD, such as obsessive-compulsive disorder and parenting strategies, were not exclusively investigated. The mixture of adolescent patients who likely remit or persist in the future also limits our power to discover the GM patterns associated with disease persistence. Future investigations leveraging longitudinal information of patient disease progression are necessary to verify and refine current findings.

\section{Acknowledgements}

We would like to thank the supporting agency of the study, the National Institutes of Health, through the grant 1 R01MH106655. M.H. is supported by a personal Veni grant from the Netherlands Organization for Scientific Research (NWO, grant number 91619115). This study makes use of data from the Dutch NeuroIMAGE project and the Dutch site of IMpACT (International Multi-center persistent ADHD Collaboration) project. The data are available through the collaboration with NeurolMAGE project (https://www.ru.nl/donders/vm-site/ collaborations/projects/neuroimage/), and with IMpACT (https://www.ru.nl/ donders/vm-site/collaborations/projects/impact/). The NeurolMAGE project was supported by NWO Large Investment grant 1750102007010 (Dr Buitelaar), ZonMW Addiction: Risk Behaviour and Dependency Grant 60-60600-97-193 (Dr Buitelaar), NWO Brain \& Cognition: an Integrative Approach grant 433-09-242 (Dr Buitelaar), NWO National Initiative Brain \& Cognition 056-13-015 (Dr Buitelaar), the EU FP7 grants TACTICS (278948), IMAGEMEND (602450), MATRICS (603016) and AGGRESSOTYPE (602805), EU IMI grant EU-AIMS (115300) and EU H2020 grant Eat2beNICE (728018) and grants from Radboudumc, University Medical Center Groningen, Accare, and VU University Amsterdam. The Dutch IMpACT study acknowledges the following sources of support: The Netherlands Organization for Scientific Research (NWO), i.e., the NWO Brain \& Cognition Excellence Program (grant 433-09-229) and the Vici Innovation Program (grant 016-130-669 to BF). Additional support was received from the European Community's Seventh Framework Programme (FP7/2007-2013) under grant agreements n 602805 (Aggressotype), $n^{\circ} 602450$ (IMAGEMEND), and $n^{\circ} 278948$ (TACTICS) as well as from the European Community's Horizon 2020 Programme (H2020/2014-2020) under grant agreements $n^{\circ} 643051$ (MiND), Eat2beNICE (728018) and no 667302 (CoCA). The work was also supported by grants for the ENIGMA Consortium (grant number U54 EB020403) from the BD2K Initiative of a cross-NIH partnership and by the ECNP Network ADHD across the Lifespan. The authors also thank all participants to these two projects. 


\section{Author details}

${ }^{1}$ Tri-institutional Center for Translational Research in Neuroimaging and Data Science (TReNDS), Georgia State University Georgia Institute of Technology and Emory University, Atlanta, GA, USA. ${ }^{2}$ School of Electrical and Computer Engineering, Georgia Institute of Technology, Atlanta, GA, USA. ${ }^{3}$ Department of Psychology, Georgia State University, Atlanta, GA, USA. ${ }^{4}$ Department of Psychiatry, Donders Institute for Brain, Cognition and Behaviour, Radboud University Medical Center, Nijmegen, The Netherlands. ${ }^{5}$ Department of Human Genetics, Donders Institute for Brain, Cognition and Behaviour, Radboud University Medical Center, Nijmegen, The Netherlands. ${ }^{6} \mathrm{Clinical}$ Neuropsychology Section, Vrije Universiteit Amsterdam, Amsterdam, The Netherlands. "Emma Children's Hospital, Amsterdam UMC, University of Amsterdam, Amsterdam, The Netherlands. ${ }^{8}$ Department of Psychiatry, University of Groningen, Groningen, The Netherlands. ${ }^{9}$ Faculty of Behavioral and Movement Sciences, Vrije Universiteit Amsterdam, Amsterdam, The Netherlands. ${ }^{10}$ Department of Computer Science, Georgia State University, Atlanta, GA, USA

\section{Conflict of interest}

J.K.B. has been in the past 3 years a consultant to/member of advisory board of/and/or speaker for Janssen Cilag BV, Eli Lilly, Lundbeck, Shire, Roche, Medice, Novartis, and Servier. He has received research support from Roche and Vifor. $\mathrm{He}$ is not an employee of any of these companies, and not a stock shareholder of any of these companies. He has no other financial or material support, including expert testimony, patents, royalties. The remaining authors declare no competing interests.

\section{Publisher's note}

Springer Nature remains neutral with regard to jurisdictional claims in published maps and institutional affiliations.

Supplementary information The online version contains supplementary material available at https://doi.org/10.1038/s41398-021-01301-1.

Received: 2 April 2020 Revised: 14 February 2021 Accepted: 2 March 2021 Published online: 25 March 2021

\section{References}

1. Faraone, S. V., Biederman, J. \& Mick, E. The age-dependent decline of attention deficit hyperactivity disorder: a meta-analysis of follow-up studies. Psychol. Med. 36, 159-165 (2006).

2. Barkley, R. A., Fischer, M., Smallish, L. \& Fletcher, K. The persistence of attentiondeficit/hyperactivity disorder into young adulthood as a function of reporting source and definition of disorder. J. Abnorm. Psychol. 111, 279-289 (2002).

3. Simon, V., Czobor, P., Balint, S., Meszaros, A. \& Bitter, I. Prevalence and correlates of adult attention-deficit hyperactivity disorder: meta-analysis. Br. J. Psychiatry 194, 204-211 (2009).

4. Fayyad, J. et al. Cross-national prevalence and correlates of adult attentiondeficit hyperactivity disorder. Br. J. Psychiatry 190, 402-409 (2007).

5. American Psychiatric Association. Diagnostic and Statistical Manual of Mental Disorders 5th edn. (American Psychiatric Association, 2013).

6. Luo, Y., Weibman, D., Halperin, J. M. \& Li, X. A review of heterogeneity in attention deficit/hyperactivity disorder (ADHD). Front. Hum. Neurosci. 13, 42 (2019).

7. Spencer, T. J., Biederman, J. \& Mick, E. Attention-deficit/hyperactivity disorder: diagnosis, lifespan, comorbidities, and neurobiology. J. Pediatr. Psychol. 32 631-642 (2007).

8. Faraone, S. V. et al. Attention-deficit/hyperactivity disorder. Nat. Rev. Dis. Prim. 1, 15020 (2015).

9. Steinhausen, H. C. The heterogeneity of causes and courses of attentiondeficit/hyperactivity disorder. Acta Psychiatr. Scand. 120, 392-399 (2009).

10. Kooij, S. J. et al. European consensus statement on diagnosis and treatment of adult ADHD: The European Network Adult ADHD. BMC Psychiatry 10, 67 (2010).

11. Kooij, J. J. S. et al. Updated European Consensus Statement on diagnosis and treatment of adult ADHD. Eur. Psychiatry 56, 14-34 (2019).

12. Wodushek, T. R. \& Neumann, C. S. Inhibitory capacity in adults with symptoms of Attention Deficit/Hyperactivity Disorder (ADHD). Arch. Clin. Neuropsychol. 18, 317-330 (2003).
13. Mostert, J. C. et al. Cognitive heterogeneity in adult attention deficit/hyperactivity disorder: a systematic analysis of neuropsychological measurements. Eur. Neuropsychopharmacol. 25, 2062-2074 (2015).

14. Willcutt, E. G., Doyle, A. E., Nigg, J. T., Faraone, S. V. \& Pennington, B. F. Validity of the executive function theory of attention-deficit/hyperactivity disorder: a meta-analytic review. Biol. Psychiatry 57, 1336-1346 (2005).

15. Mueller, A., Hong, D. S., Shepard, S. \& Moore, T. Linking ADHD to the neural circuitry of attention. Trends Cogn. Sci. 21, 474-488 (2017).

16. Martinussen, R., Hayden, J., Hogg-Johnson, S. \& Tannock, R. A meta-analysis of working memory impairments in children with attention-deficit/hyperactivity disorder. J. Am. Acad. Child Adolesc. Psychiatry 44, 377-384 (2005).

17. Forslund, T., Brocki, K. C., Bohlin, G., Granqvist, P. \& Eninger, L. The heterogeneity of attention-deficit/hyperactivity disorder symptoms and conduct problems: cognitive inhibition, emotion regulation, emotionality, and disorganized attachment. Br. J. Dev. Psychol. 34, 371-387 (2016).

18. Slaats-Willemse, D., Swaab-Barneveld, H., de Sonneville, L., van der Meulen, E. \& Buitelaar, J. Deficient response inhibition as a cognitive endophenotype of ADHD. J. Am. Acad. Child Adolesc. Psychiatry 42, 1242-1248 (2003).

19. Rommelse, N. N. et al. Are motor inhibition and cognitive flexibility dead ends in ADHD? J. Abnorm. Child Psychol. 35, 957-967 (2007)

20. Alderson, R. M., Hudec, K. L., Patros, C. H. \& Kasper, L. J. Working memon deficits in adults with attention-deficit/hyperactivity disorder (ADHD): an examination of central executive and storage/rehearsal processes. J. Abnorm. Psychol. 122, 532-541 (2013).

21. Mostert, J. C. et al. Similar subgroups based on cognitive performance parse heterogeneity in adults with ADHD and healthy controls. J. Atten. Disord. 22, 281-292 (2018).

22. Coghill, D. R., Seth, S. \& Matthews, K. A comprehensive assessment of memory, delay aversion, timing, inhibition, decision making and variability in attention deficit hyperactivity disorder: advancing beyond the three-pathway models. Psychol. Med. 44, 1989-2001 (2014).

23. Jiang, W. et al. Structural brain alterations and their association with cognitive function and symptoms in Attention-deficit/Hyperactivity Disorder families. Neuroimage Clin. 27, 102273 (2020).

24. Duan, $K$. et al. Neural correlates of cognitive function and symptoms in attention-deficit/hyperactivity disorder in adults. Neuroimage Clin. 19, 374-383 (2018).

25. Frodl, T. \& Skokauskas, N. Meta-analysis of structural MRI studies in children and adults with attention deficit hyperactivity disorder indicates treatment effects. Acta Psychiatr. Scand. 125, 114-126 (2012).

26. Tremols, V. et al. Differential abnormalities of the head and body of the caudate nucleus in attention deficit-hyperactivity disorder. Psychiatry Res. 163, 270-278 (2008).

27. Perlov, E. et al. Hippocampus and amygdala morphology in adults with attention-deficit hyperactivity disorder. J. Psychiatry Neurosci. 33, 509-515 (2008).

28. Valera, E. M., Faraone, S. V., Murray, K. E. \& Seidman, L. J. Meta-analysis of structural imaging findings in attention-deficit/hyperactivity disorder. Biol. Psychiatry 61, 1361-1369 (2007).

29. Curatolo, P., Paloscia, C., D'Agati, E., Moavero, R. \& Pasini, A. The neurobiology of attention deficit/hyperactivity disorder. Eur. J. Paediatr. Neurol. 13, 299-304 (2009).

30. Seidman, L. J. et al. Gray matter alterations in adults with attention-deficit/ hyperactivity disorder identified by voxel based morphometry. Biol. Psychiatry 69, 857-866 (2011).

31. Castellanos, F. X. et al. Developmental trajectories of brain volume abnormalities in children and adolescents with attention-deficit/hyperactivity disorder. JAMA 288, 1740-1748 (2002).

32. Proal, E. et al. Brain gray matter deficits at 33-year follow-up in adults with attention-deficit/hyperactivity disorder established in childhood. Arch. Gen. Psychiatry 68, 1122-1134 (2011).

33. Makris, N. et al. Toward defining the neural substrates of ADHD: a controlled structural MRI study in medication-naive adults. J. Atten. Disord. 19, 944-953 (2015).

34. Roman-Urrestarazu, A. et al. Brain structural deficits and working memory fMRI dysfunction in young adults who were diagnosed with ADHD in adolescence. Eur. Child Adolesc. Psychiatry 25, 529-538 (2016).

35. Maier, S. et al. Discrete global but no focal gray matter volume reductions in unmedicated adult patients with attention-deficit/hyperactivity disorder. Biol. Psychiatry 80, 905-915 (2015). 
36. Jacobson, L. A. et al. Anomalous brain development is evident in preschoolers with attention-deficit/hyperactivity disorder. J. Int. Neuropsychol. Soc. 24 531-539 (2018).

37. Castellanos, F. X. \& Proal, E. Large-scale brain systems in ADHD: beyond the prefrontal-striatal model. Trends Cogn. Sci. 16, 17-26 (2012).

38. Greven, C. U. et al. Developmentally stable whole-brain volume reductions and developmentally sensitive caudate and putamen volume alterations in those with attention-deficit/hyperactivity disorder and their unaffected siblings. JAMA Psychiatry 72, 490-499 (2015).

39. Sudre, G., Mangalmurti, A. \& Shaw, P. Growing out of attention deficit hyperactivity disorder: insights from the 'remitted' brain. Neurosci. Biobehav. Rev. 94, 198-209 (2018).

40. Gupta, C. N. et al. Patterns of gray matter abnormalities in schizophrenia based on an international mega-analysis. Schizophr. Bull. 41, 1133-1142 (2015).

41. Caprihan, A. et al. Source-based morphometry analysis of group differences in fractional anisotropy in schizophrenia. Brain Connect. 1, 133-145 (2011).

42. Xu, L., Groth, K. M., Pearlson, G., Schretlen, D. J. \& Calhoun, V. D. Source-based morphometry: the use of independent component analysis to identify gray matter differences with application to schizophrenia. Hum. Brain Mapp. $\mathbf{3 0}$ 711-724 (2009)

43. Calhoun, V. D., Liu, J. \& Adall, T. A review of group ICA for fMRI data and ICA for joint inference of imaging, genetic, and ERP data. Neuroimage 45, S163-S172 (2009).

44. Allen, E. A. et al. A baseline for the multivariate comparison of resting-state networks. Front. Syst. Neurosci. 5, 2 (2011).

45. Espinoza, F. A. et al. Whole-brain connectivity in a large study of Huntington's disease gene mutation carriers and healthy controls. Brain Connect. 8, 166-178 (2018).

46. Onnink, A. M. et al. Brain alterations in adult ADHD: effects of gender, treatment and comorbid depression. Eur. Neuropsychopharmacol. 24, 397-409 (2014).

47. von Rhein, D. et al. The NeurolMAGE study: a prospective phenotypic, cognitive, genetic and MRI study in children with attention-deficit/hyperactivity disorder. Design and descriptives. Eur. Child Adolesc. Psychiatry 24, 265-281 (2015).

48. Wechsler, D., Van der Steene, G., Vertommen, H., Bleichrodt, N. \& Uiterwijk, J. WAIS-III: Nederlandstalige bewerking (Swets and Harcourt Test Publishers, 2000).

49. Esteban, O. et al. MRIQC: Advancing the automatic prediction of image quality in MRI from unseen sites. PLOS ONE 12, e0184661 (2017).

50. Wilke, M., Holland, S. K. Altaye, M. \& Gaser, C. Template-O-Matic: a toolbox for creating customized pediatric templates. Neuroimage 41, 903-913 (2008).

51. Dennis, M. et al. Why IQ is not a covariate in cognitive studies of neurodevelopmental disorders. J. Int. Neuropsychol. Soc. 15, 331-343 (2009).

52. Giedd, J. N. et al. Brain development during childhood and adolescence: a longitudinal MRI study. Nat. Neurosci. 2, 861-863 (1999).

53. Hoogman, M. et al. Subcortical brain volume differences in participants with attention deficit hyperactivity disorder in children and adults: a cross-sectional mega-analysis. Lancet Psychiatry 4, 310-319 (2017).

54. Morris, L. S. et al. Fronto-striatal organization: defining functional and microstructural substrates of behavioural flexibility. Cortex 74, 118-133 (2016).

55. Landau, S. M., Lal, R., O'Neil, J. P., Baker, S. \& Jagust, W. J. Striatal dopamine and working memory. Cereb. Cortex 19, 445-454 (2009).

56. Massat, l. et al. Working memory-related functional brain patterns in never medicated children with ADHD. PLoS ONE 7, e49392 (2012).

57. Baddeley, A. Working memory: theories, models, and controversies. Annu. Rev. Psychol. 63, 1-29 (2012).

58. Funahashi, S. Working memory in the prefrontal cortex. Brain Sci. 7, 49 (2017).

59. Murray, J. D. et al. Stable population coding for working memory coexists with heterogeneous neural dynamics in prefrontal cortex. Proc. Natl Acad. Sci. USA 114, 394-399 (2017).

60. Eriksson, J., Vogel, E. K., Lansner, A., Bergstrom, F. \& Nyberg, L. Neurocognitive architecture of working memory. Neuron $\mathbf{8 8}, 33-46$ (2015).
61. Goghari, V. M., MacDonald, A. W. \& Sponheim, S. R. Relationship between prefrontal gray matter volumes and working memory performance in schizophrenia: a family study. Schizophr. Res. 153, 113-121 (2014).

62. Takeuchi, $\mathrm{H}$. et al. Global associations between regional gray matter volume and diverse complex cognitive functions: evidence from a large sample study. Sci. Rep. 7, 10014 (2017).

63. Biederman, J. et al. Are cognitive deficits in attention deficit/hyperactivity disorder related to the course of the disorder? A prospective controlled follow-up study of grown up boys with persistent and remitting course. Psychiatry Res. 170, 177-182 (2009).

64. Berquin, P. C. et al. Cerebellum in attention-deficit hyperactivity disorder: a morphometric MRI study. Neurology 50, 1087-1093 (1998).

65. Emond, V., Joyal, C. \& Poissant, H. Structural and functional neuroanatomy of attention-deficit hyperactivity disorder (ADHD). Encephale 35, 107-114 (2009).

66. Carmona, S. et al. Global and regional gray matter reductions in ADHD: a voxel-based morphometric study. Neurosci. Lett. 389, 88-93 (2005).

67. Schmahmann, J. D. The role of the cerebellum in cognition and emotion: personal reflections since 1982 on the dysmetria of thought hypothesis, and its historical evolution from theory to therapy. Neuropsychol. Rev. 20, 236-260 (2010).

68. Schmahmann, J. D. The cerebellum and cognition. Neurosci. Lett. 688, 62-75 (2019).

69. Gottwald, B., Wilde, B., Mihajlovic, Z. \& Mehdorn, H. M. Evidence for distinct cognitive deficits after focal cerebellar lesions. J. Neurol. Neurosurg. Psychiatry 75, 1524-1531 (2004).

70. Stoodley, C. J. \& Schmahmann, J. D. Functional topography in the human cerebellum: a meta-analysis of neuroimaging studies. Neuroimage 44, 489-501 (2009).

71. Middleton, F. A. \& Strick, P. L. Cerebellar output: motor and cognitive channels. Trends Cogn. Sci. 2, 348-354 (1998).

72. Engelhardt, E., Moreira, D. M. \& Laks, J. Cerebrocerebellar system and Arnold's bundle-a tractographic study: preliminary results. Dement. Neuropsychol. 4, 293-299 (2010).

73. Kellermann, T. et al. Effective connectivity of the human cerebellum during visual attention. J. Neurosci. 32, 11453-11460 (2012).

74. Buckner, R. L., Krienen, F. M., Castellanos, A., Diaz, J. C. \& Yeo, B. T. The organization of the human cerebellum estimated by intrinsic functional connectivity. J. Neurophysiol. 106, 2322-2345 (2011).

75. Piek, J. P., Pitcher, T. M. \& Hay, D. A. Motor coordination and kinaesthesis in boys with attention deficit-hyperactivity disorder. Dev. Med. Child. Neurol. 41, 159-165 (1999).

76. Patankar, V. C., Sangle, J. P., Shah, H. R., Dave, M. \& Kamath, R. M. Neurological soft signs in children with attention deficit hyperactivity disorder. Indian J. Psychiatry 54, 159-165 (2012).

77. Koziol, L. F., Budding, D. E. \& Chidekel, D. From movement to thought: executive function, embodied cognition, and the cerebellum. Cerebellum 11, 505-525 (2012).

78. Pitzianti, M. et al. Neurological soft signs are associated with attentional dysfunction in children with attention deficit hyperactivity disorder. Cogn. Neuropsychiatry 21, 475-493 (2016).

79. Ghaziri, J. et al. Subcortical structural connectivity of insular subregions. Sci. Rep. 8, 8596 (2018).

80. Yeterian, E. H. \& Pandya, D. N. Corticostriatal connections of the superior temporal region in rhesus monkeys. J. Comp. Neurol. 399, 384-402 (1998).

81. Hong, S. B. et al. Subthreshold attention-deficit/hyperactivity disorder is associated with functional impairments across domains: a comprehensive analysis in a large-scale community study. Eur. Child Adolesc. Psychiatry 23, 627-636 (2014)

82. Faraone, S. V. et al. Diagnosing adult attention deficit hyperactivity disorder: are late onset and subthreshold diagnoses valid? Am. J. Psychiatry 163, 1720-1729 (2006).

83. Kirova, A. M. et al. Are subsyndromal manifestations of attention deficit hyperactivity disorder morbid in children? A systematic qualitative review of the literature with meta-analysis. Psychiatry Res. 274, 75-90 (2019). 